\title{
Mapping Atomic Motions with Ultrabright Electrons: Fundamental Space-Time Limits to Imaging Chemistry and Biological Processes
}

\author{
R. J. Dwayne Miller
}

Departments of Physics and Chemistry, University of Toronto, Toronto, ON, Canada, United States

One of the grand challenges in science has been to watch atomic motions on the primary timescales of chemistry(1). This prospect would provide a direct observation of the reaction forces, the very essence of chemistry, and the central unifying concept of transition states that links chemistry to biology. This experiment has been referred to as "making the molecular movie" with respect to observing net rms atomic motions during structural changes. There are not only extraordinary requirements for simultaneous spatialtemporal resolution but equally important, due to sample limitations, also one on source brightness. Taking the problem to be an imaging problem, as one makes the shutter speed shorter and shorter, a brighter and brighter source is needed to maintain image contrast. With the development of ultrabright electron capable of literally lighting up atomic motions, this experiment was first realized (1-3) and efforts accelerated with the onset of XFELs (4). A number of different chemical reactions will be discussed from electrocyclization with conserved stereochemistry(5), intermolecular electron transfer for organic systems $(6,7)$, metal to metal electron transfer $(8)$, to the recent observation of coherently directed bond formation using the classic $\mathrm{I}_{3}{ }^{-}$system, in a process analogous to a quantum Newton's cradle (9). These studies have discovered that these nominally 100+ dimensional problems, representing the number of degrees of freedom in the system, distilled down to atomic projections along a few principle reaction coordinates $(1$, $3,10)$. The specific details depend on the spatial resolution to these motions. The most dramatic example will be shown for the simplest form of chemistry, electron transfer, which is the heart of redox chemistry. No bonds are made or broken. The reaction coordinate is dictated by medium repolarization that stabilizes the charge transfer state. This motion was captured with sub- $\AA$ (.01 $\AA$ ) and $100 \mathrm{fs}$ timescale resolution the fundamental space-time resolution to following the primary processes of chemistry $(8,11)$. Without any detailed analysis, the key large-amplitude modes can be identified by eye from the molecular movie. This reduction in dimensionality appears to be general, arising from the very strong anharmonicity of the many body potential in the barrier crossing region. We now are beginning to see the underlying physics for the generalized reaction mechanisms that have been empirically discovered over time. The "magic of chemistry" is this enormous reduction in dimensionality in the barrier crossing region that ultimately makes chemical concepts transferrable. How far can this reductionist view be extended with respect to complexity? The ultimate goal in scaling system complexity is to obtain atomically resolved protein functions to understand how nature tamed chemistry over all conceivable length scales. New approaches based on the prinicples used for femtosecond electron diffraction, both in terms of source technology and image reconstruction, hold promise for real space studies of single biomolecules. This prospect is within reach and will provide a definitive test of the collective mode coupling model (12) to bridge chemistry to biology, which will be discussed as the driving force for this work.

\section{References}

1. R. J. D. Miller, Mapping Atomic Motions with Ultrabright Electrons: The Chemists' Gedanken Experiment Enters the Lab Frame. Annual Review of Physical Chemistry. 65, 583-604 (2014).

2. $\quad$ B. J. Siwick, J. R. Dwyer, R. E. Jordan, R. J. D. Miller, An Atomic-Level View of Melting Using Femtosecond Electron Diffraction. Science. 302, 1382-1385 (2003).

3. A. A. Ischenko, P. M. Weber, R. J. D. Miller, Capturing Chemistry in Action with Electrons: Realization of Atomically Resolved Reaction Dynamics. Chemical Reviews. 117, 11066-11124 (2017). 
4. R. J. D. Miller, Femtosecond Crystallography with Ultrabright Electrons and X-rays: Capturing Chemistry in Action. Science. 343, 1108-1116 (2014).

5. H. Jean-Ruel, M. Gao, M. A. Kochman, C. Lu, L. C. Liu, R. R. Cooney, C. A. Morrison, R. J. D. Miller, Ring-Closing Reaction in Diarylethene Captured by Femtosecond Electron Crystallography. The Journal of Physical Chemistry B. 117, 15894-15902 (2013).

6. M. Gao, et al., Mapping molecular motions leading to charge delocalization with ultrabright electrons. Nature. 496, 343-346 (2013).

7. L. C. Liu et al., Ultrafast electron diffraction study of single-crystal (EDO-TTF) 2 SbF 6 : Counterion effect and dimensionality reduction. Chemical Physics Letters. 683, 160-165 (2017).

8. T. Ishikawa et al. , Direct observation of collective modes coupled to molecular orbital-driven charge transfer. Science. 350, 1501-1505 (2015).

9. R. Xian, G. Corthey, D. M. Rogers, C. A. Morrison, V. I. Prokhorenko, S. A. Hayes, R. J. D. Miller, Coherent ultrafast lattice-directed reaction dynamics of triiodide anion photodissociation. Nature Chemistry. 9, 516-522 (2017).

10. Z. Li, S. Gyawali, A. A. Ischenko, S. Hayes, R. J. D. Miller, Mapping Atomic Motions with Electrons: Toward the Quantum Limit to Imaging Chemistry. ACS Photonics. 7, 296-320 (2020).

11. R. J. D. Miller, Energetics and Dynamics of Deterministic Protein Motion. Accounts of Chemical Research. 27, 145150 (1994). 